\title{
Pure ground glass nodular adenocarcinomas: Are preoperative positron emission tomography/computed tomography and brain magnetic resonance imaging useful or necessary?
}

\author{
Hyoun Cho, MD, ${ }^{\mathrm{a}}$ Ho Yun Lee, MD, ${ }^{\mathrm{a}}$ Jhingook Kim, MD, ${ }^{\mathrm{b}}$ Hong Kwan Kim, MD, ${ }^{\mathrm{b}}$ Joon Young Choi, MD, \\ Sang-Won Um, MD, ${ }^{\mathrm{d}}$ and Kyung Soo Lee, $\mathrm{MD}^{\mathrm{a}}$
}

\begin{abstract}
Objective: The utility of ${ }^{18} \mathrm{~F}$-Fluorodeoxyglucose positron emission tomography/ computed tomography (FDG PET/CT) scanning and brain magnetic resonance imaging (MRI) as a staging workup for lung adenocarcinoma manifesting as pure ground glass opacity (GGO) is unknown. The purpose of this study was to determine the utility of these 2 tests for preoperative staging of pure GGO nodular lung adenocarcinoma.
\end{abstract}

Methods: The study included 164 patients (male:female, 73:91; mean age, 62 years) with pure GGO nodular lung adenocarcinoma who underwent PET/CT (in 136 patients) and/or brain MRI (in 109 patients) before surgery. Pathologic $\mathrm{N}$ staging and dedicated standard imaging or follow-up imaging findings for $\mathrm{M}$ staging were used as reference standards. The median follow-up time was 47.9 months.

Results: On PET/CT scan, abnormal FDG uptake of lymph nodes was found in 2 of 136 patients (1.5\%); both were negative on final pathology. Abnormal FDG uptake of the liver was detected in 1 patient, which was also confirmed to be negative by dedicated abdominal CT. The sensitivity, specificity, positive predictive value, negative predictive value, and accuracy of PET/CT in detecting metastases were not applicable, $98 \%$ (95\% confidence interval [CI], 94\%-100\%), $0 \%(95 \% \mathrm{CI}$, 0\%-71\%), 100\% (95\% CI, 97\%-100\%), and 98\% (95\% CI, 94\%-100\%), respectively. No brain metastasis was found in preoperative brain MRI of 109 patients. Of 109 patients, $1(0.9 \%)$ developed brain metastasis 30 months after surgical resection.

Conclusions: PET/CT and brain MRI is not necessary in the staging of pure GGO nodular lung adenocarcinoma. (J Thorac Cardiovasc Surg 2015;150:514-20)

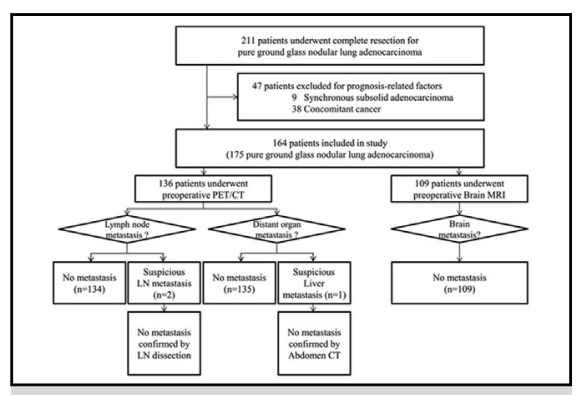

Flow chart of patient enrollment and staging workup for patients with pure ground glass opacity nodular lung adenocarcinomas.

Central Message
Patients with pure GGO adenocarcinomas had
negative PET/CT and brain MRI. Unlike guide-
lines for NSCLC, these studies are unnecessary.
Perspective
The necessity of PET/CT and brain MRI
in patients with GGO's are not determined.
But, these are routinely practiced in patients
with the adenocarcinoma manifesting as pure
GGOs. We concluded that PET/CT and brain
MRI add little utility in the staging of pure
GGO adenocarcinoma regardless of tumor
size. Our study can support the potential cost
savings, as well as reduction of unnecessary
radiation.

See Editorial Commentary page 521.

\begin{abstract}
${ }^{18}$ Fluorodeoxyglucose positron emission and computed tomography (FDG PET/CT) is recommended by the European Society of Thoracic Surgeons and American College of Chest Physicians for the preoperative (clinical) evaluation of mediastinal and distant metastases of patients with non-small cell lung cancer (NSCLS). ${ }^{1,2}$ Despite the shortcomings of FDG PET/CT brain imaging, there are no recommendations for preoperative brain magnetic resonance imaging (MRI), even though the use of MRI is

\footnotetext{
From the ${ }^{\mathrm{a} D e p a r t m e n t}$ of Radiology and Center for Imaging Science, ${ }^{\mathrm{b}}$ Department of Thoracic Surgery and Cardiovascular Surgery, ${ }^{\mathrm{c}}$ Department of Nuclear Medicine, and ${ }^{\mathrm{d} D i v i s i o n}$ of Pulmonary and Critical Care Medicine, Department of Medicine, Samsung Medical Center, Sungkyunkwan University School of Medicine, Seoul, Korea.

Received for publication Sept 11, 2014; revisions received March 10, 2015; accepted for publication March 25, 2015; available ahead of print July 15, 2015.
}

associated with better control of neurologic manifestations and longer survival. ${ }^{3-5}$ Further uncertainty is added in those patients with the earliest of adenocarcinomas; that is, those manifesting as ground glass opacities (GGOs) although there are some guidelines for clinical T1a NSCLC or stage I NSCLC. ${ }^{6-11}$ The purposes of our study were to assess the usefulness and necessity of FDG PET/CT and brain MRI in patients with GGOs and thus determine if these studies are mandatory in preoperative evaluations.

\footnotetext{
Address for reprints: Ho Yun Lee, MD, Department of Radiology and Center for Imaging Science, Samsung Medical Center, Sungkyunkwan University School of Medicine, 50, Ilwon-Dong, Kangnam-Ku, Seoul 135-710, Korea (E-mail: hoyunlee96@gmail.com). $0022-5223 / \$ 36.00$

Copyright () 2015 by The American Association for Thoracic Surgery http://dx.doi.org/10.1016/j.jtcvs.2015.06.024
} 


\section{Abbreviations and Acronyms \\ AIS = adenocarcinoma in situ \\ $\mathrm{CT}=$ computed tomography \\ FDG = fluorodeoxyglucose \\ GGN = ground glass opacity nodule \\ GGO = ground glass opacity \\ $\mathrm{LN}=$ lymph node \\ MIA = minimally invasive adenocarcinoma \\ MRI = magnetic resonance imaging \\ NSCLC $=$ non-small cell lung cancer \\ PET = positron emission tomography \\ SUVmax $=$ maximum standardized uptake value \\ TMN = tumor, node, and metastasis \\ VATS $=$ video-assisted thoracoscopic surgery}

\section{PATIENTS AND METHODS}

This retrospective study was approved by the institutional review board of Samsung Medical Center (Seoul, Republic of Korea), which waived the requirement for informed consent.

\section{Patients}

A total of 211 patients with histopathologically determined adenocarcinoma of the lung by complete surgical resection were enrolled from the thoracic surgery registry of NSCLC (October 2003-February 2014). The selection criteria for this study were available thin-section chest CT that indicated pure GGO nodules (GGNs) through the consensus review of 2 radiologists with 12 and 5 years' experience in chest CT, respectively, at the Samsung Medical Center. Pure GGNs were defined on chest CT scan because those composed purely of GGO on lung window images with no or barely identifiable soft-tissue attenuation within the lesion on mediastinal window images.

Of these 211 patients, 47 were excluded for the following reasons: 9 patients had concomitant solid or part solid adenocarcinoma in multiple nodules, including pure GGO nodular adenocarcinoma, and 38 had a previous or concurrent malignancy. Finally, 164 patients (male to female ratio, 73:91; mean age, 62 years) with pure GGO nodular lung adenocarcinoma who underwent contrast-enhanced CT before surgery were included in the analysis.

\section{Characteristics of the Study Patients}

Patient demographics and radiologic findings for the total study population are summarized in Table 1. Among the 164 patients, 175 GGNs (17 [10\%] central location; 158 [90\%] peripheral location) were identified on the screening chest CT performed at the time of initial operation. The median size of GGNs was $16.5 \mathrm{~mm}$ (range, 4-35 mm). Tumors that were larger than $30 \mathrm{~mm}$ were all negative for lymph node (LN) metastasis and distant metastasis. LN staging was determined using the pathologic results from video-assisted thoracoscopic surgery (VATS) $(\mathrm{n}=105)$, thoracotomy $(n=6)$, and robot-assisted lobectomy $(n=2)$. The mean extracted $L N$ station number per operation was 4.1 , which were all pathologically negative of metastasis.

Of these 164 patients, imaging data were available for 136 who had FDG-PET/CT and 109 who had brain MRI before surgery (Figure 1). Twenty-eight patients did not undergo preoperative FDG-PET/CT; these patients had 31 pure GGNs. Among them, 28 pure GGNs (90\%) were 2 $\mathrm{cm}$ or smaller, and the other $3(10 \%)$ were $\leq 3 \mathrm{~cm}$ and clinical stage T1b lung cancer. Contrast-enhanced CT of these 28 patients revealed no LN enlargement. Therefore, for these 28 patients, metastasis determined using clinical and contrast-enhanced CT imaging studies served as the reference standard for determining the absence of metastasis. Fifty-five patients did not undergo brain MRI; 58 pure GGNs of these 55 patients were $\leq 3 \mathrm{~cm}$ in size and all were stage I lung adenocarcinoma. There was no evidence of LN metastasis or distant metastasis during the follow-up period (47.9 \pm 23.1 months). Survival rate was $100 \%$.

All pure GGNs except for 1 case were pathologic stage 1A. Pathologic tumor, node, and metastasis (TNM) staging was T1a to T3 N0 M0. The pathology is summarized in Table 2. Of 175 tumors in 164 patients, 34 were adenocarcinoma in situ (AIS) (19\%), 54 were minimally invasive adenocarcinoma (MIA) $(31 \%)$, and 87 were invasive adenocarcinoma $(50 \%)$. The histologic subtypes, in decreasing order, were lepidic $(69 \%)$, acinar $(26 \%)$, and papillary $(5 \%)$. All tumors were negative for lymphatic or vascular invasion. Even among nonlepidic adenocarcinoma subtypes such as acinar and papillary, there was no LN metastasis or distant metastasis. In 1 case the tumor invaded into the parietal pleura of the chest wall, and was TNM stage T3 and stage 2B (tumor size, $21.9 \mathrm{~mm}$; histologic subtype, acinar predominant). LN metastasis or distant metastasis was not found in this patient.

\section{Preoperative Staging with Imaging}

The presence of LN metastases was evaluated using chest CT and FDG PET/CT. Metastases in other organs were determined by brain MRI and FDG PET/CT. All positive results were based on preoperative reports and also reviewed again retrospectively.

The following CT scanners were used in this study: a second-generation dual-source CT system (Somatom Definition Flash, Siemens Healthcare, Erlangen, Germany [7 studies]), a 64-MDCT scanner (Aquilion 64, Toshiba Medical Systems, Otawara, Japan [46 studies] or LightSpeed VCT, GE Healthcare [57 studies], Milwaukee, Wisc), a 40-MDCT scanner (Brilliance 40, Philips Healthcare, Best, The Netherlands [32 studies]), a 16-MDCT scanner (LightSpeed 16, GE Healthcare [36 studies] or LightSpeed QX/i, GE Healthcare [seven studies]), and an 8-MDCT scanner (LightSpeed Ultra, GE Healthcare [17 studies]). Unenhanced CT images were obtained with the following parameters: $120 \mathrm{kVp}$; 100 to $250 \mathrm{~mA}$; beam pitch, 0.875 to 1.675 ; and section thickness, 0.75 to $2.5 \mathrm{~mm}$ for transverse. All imaging data were reconstructed with a bone (high-frequency) algorithm.

For FDG PET/CT scan, patients received an intravenous injection of 5.5 $\mathrm{MBq} / \mathrm{kg}$ FDG and then rested for 60 minutes before scanning. Images were acquired using a PET-CT device (primarily a Discovery STe scanner; GE Healthcare), which included a PET scanner (2.5 minutes per frame in a 3-dimensional mode) and a 16-slice CT scanner.

Brain MRI was conducted using a 1.5-T and 3-T MRI scanner (Achieva; Philips Medical Systems, Best, the Netherlands) with a standard head coil. Three sequences were used for imaging: a T2W, axial, turbo spin-echo pulse sequence with fat suppression; a fluid-attenuation, inversionrecovery spin-echo sequence; and a noncontrast-enhanced and a contrastenhanced T1W spin-echo sequence.

Two radiologists (with 12 and 5 years' experience in chest CT, respectively) evaluated the chest CT scans in consensus. LN assessment was based on LN size on the chest CT, with a short axis diameter $>10 \mathrm{~mm}$ defined as abnormal. ${ }^{2}$ If mediastinal or hilar nodes showed high attenuation (>70 Hounsfield units) or benign calcification (eg, central nodular, laminated, popcorn, or diffuse) on unenhanced $\mathrm{CT}$ images, they were regarded as benign irrespective of their size. ${ }^{12,13}$

A chest radiologist with 5 years' experience in chest $\mathrm{CT}$ and a nuclear medicine physician with 20 years' experience of PET/CT interpretation evaluated the integrated FDG PET/CT images independently, and all decisions were reached in consensus. Both clinicians were unaware of the results of clinical and pathologic evaluation. On FDG PET/CT images, all LNs in the thorax with FDG uptake no greater than the normal background activity of the mediastinal blood pool (which had a maximum standardized uptake value $[\mathrm{SUVmax}]<1.5$, regardless of size) were considered cN0. An 
TABLE 1. Demographic data and characteristics of 164 patients with pure ground glass opacity nodular lung adenocarcinomas

\begin{tabular}{lr}
\hline \multicolumn{1}{c}{ Characteristic } & Result \\
\hline Gender & \\
Male & $73(45)$ \\
Female & $91(55)$ \\
Age, $y$ & $61.5 \pm 9.5$ \\
History of smoking & \\
$\quad$ Current smoker & $20(12)$ \\
Ex-smoker & $38(23)$ \\
Never smoker & $106(65)$ \\
Size of ground glass nodules, mm & $16.5 \pm 6.3$ \\
Ground glass nodules per patient & \\
1 & $155(95)$ \\
2 & $7(4)$ \\
3 & $2(1)$ \\
Outcome & \\
Follow-up period, mo & $47.9 \pm 23.1$ \\
Local recurrence & $2(1)$ \\
Metastasis & $1(1)$ \\
\hline
\end{tabular}

Data are presented as number of patients (\%) or median \pm standard deviation.

LN with SUVmax $\geq 1.5$ was considered "suspicious for malignancy." Abnormal focal FDG uptake accompanied by a corresponding anatomic alteration was considered indicative of distant metastasis. The presence of abnormal uptake was determined qualitatively by comparing the uptake of the lesion in question with that of a mediastinal blood pool in the thorax or that of liver uptake in the abdomen, bones, or the remaining soft tissues. $^{13,14}$

Two chest radiologists with 4 years' experience in whole-body MRI analysis, who were unaware of the clinical and PET/CT findings or histologic diagnoses, interpreted the brain MR images and made decisions on the findings by consensus. On brain MRI, metastases were considered present when an enhancing nodule or mass was observed in the brain parenchyma on contrast-enhanced T1-weighted images.

\section{Reference Standard for Metastases and Follow-up Evaluation}

The final decisions on the presence of LN metastases and distant metastases in each patient were reached based on the results of dedicated standard imaging, pathologic examination, or a follow-up examination.

A lung pathologist with 19 years' experience in lung pathology interpreted all tissue sections and LNs. The seventh edition of the American Joint Committee on Cancer TNM staging system for lung cancer was adopted for proper postoperative staging. The specimen was reviewed according to the regulations set forth in the 2011 guidelines of the International Association for the Study of Lung Cancer/ American Thoracic Society/European Respiratory Society International Multidisciplinary Lung Adenocarcinoma Classification criteria, and the most abundant subtype of existent tumor histologic subtypes (atypical adenomatous hyperplasia, AIS, MIA, and invasive adenocarcinoma of lepidic, acinar, papillary, micropapillary, and solid patterns) was reviewed for each tumor. ${ }^{15}$ The presence of lymphatic or vascular invasion was also recorded.

LN staging was determined using the pathologic results from VATS $(\mathrm{n}=105)$, thoracotomy $(\mathrm{n}=6)$, and robot-assisted lobectomy $(\mathrm{n}=2)$. The pathologic results of $\mathrm{LN}$ staging were not available for 51 patients who did not undergo LN dissection by VATS wedge resection. None of these patients had enlarged LNs in the preoperative chest CT or abnormal FDG uptake on PET/CT images. For these patients, LN metastasis determined using clinical and follow-up imaging studies over at least 6 months, including chest CT and FDG PET/CT, served as the reference standard for determining the absence of metastasis.

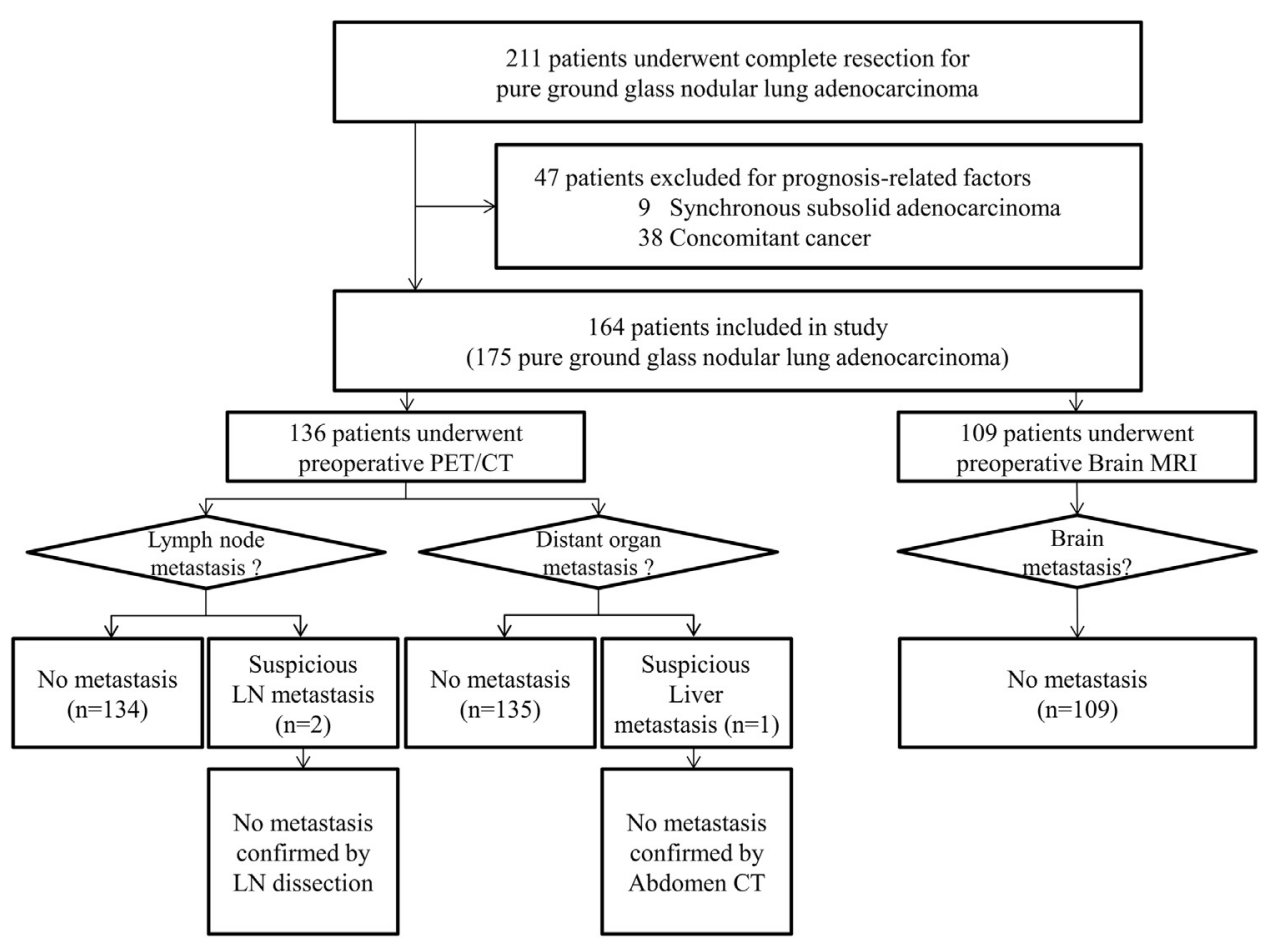

FIGURE 1. Flow chart of patient enrollment and staging workup for patients with pure ground glass opacity nodular lung adenocarcinomas. PET, Positron emission tomography; $C T$, computed tomography; $L N$, lymph node; $M R I$, magnetic resonance imaging. 
TABLE 2. Operation and pathology

\begin{tabular}{|c|c|}
\hline Variable & Result \\
\hline \multicolumn{2}{|l|}{ Surgical procedure } \\
\hline Video-assisted thoracoscopic surgery & 155 \\
\hline Open & 7 \\
\hline Robotic & 2 \\
\hline \multicolumn{2}{|l|}{ Extent of resection } \\
\hline Wedge resection & $59(36)$ \\
\hline Segmentectomy & $5(3)$ \\
\hline Lobectomy & $100(61)$ \\
\hline Tumor size in chest computed tomography scan, $\mathrm{mm}$ & $16.5 \pm 6.3$ \\
\hline$\leq 10$ & $27(15)$ \\
\hline $10-20$ & $103(59)$ \\
\hline $20-30$ & $40(23)$ \\
\hline$>30$ & $5(3)$ \\
\hline \multicolumn{2}{|l|}{ Pathologic tumor, node, and metastasis stage } \\
\hline T1a & $155(89)$ \\
\hline $\mathrm{T} 1 \mathrm{~b}$ & $18(10)$ \\
\hline $\mathrm{T} 2 \mathrm{a}$ & $1(1)$ \\
\hline $\mathrm{T} 3$ & $1(1)$ \\
\hline No & $175(100)$ \\
\hline M0 & $175(100)$ \\
\hline \multicolumn{2}{|l|}{ Histologic type } \\
\hline Adenocarcinoma in situ & $34(19)$ \\
\hline Minimally invasive adenocarcinoma & $54(31)$ \\
\hline Invasive adenocarcinoma & $87(50)$ \\
\hline Lepidic predominant & $60(69)$ \\
\hline Acinar predominant & $23(26)$ \\
\hline Papillary predominant & $4(5)$ \\
\hline
\end{tabular}

Data are presented as number of patients or nodules $(\%)$ or median \pm standard deviation.

All patients who underwent lung resections were followed-up from the day after surgery. Postoperative follow-up procedures, including a physical examination and chest radiograph every 3 months and chest and abdominal CT examinations every 6 months, were performed for the first 2 years. Thereafter, a physical examination and chest radiograph were performed every 6 months and a chest CT examination was performed annually. The mean clinical follow-up time was 47.9 months.

\section{Statistical Analysis}

Categorical variables were summarized using frequencies and percentages. Continuous variables were analyzed using the median and standard deviations. Statistical analyses were performed with MedCalc (MedCalc Software, Inc, Mariakerke, Belgium).

\section{RESULTS \\ Efficacy of PET/CT and Brain MRI for Nodal Staging and Distant Metastasis}

On PET/CT scan, abnormal FDG uptake by the LNs was found in 2 of 136 patients $(1.5 \%)$. One patient had 2 pure GGNs (tumor size $29.8 \mathrm{~mm}$ in right upper lobe and 23.5 $\mathrm{mm}$ in left lower lobe). Right supraclavicular LN, right paratracheal LN, subcarinal LN, and bilateral pulmonary hilar LNs had FDG uptake on PET/CT images (SUVmax range, 5.2-7.0), indicating multiple metastatic lymphadenopathy. The short diameter of the right paratracheal $\mathrm{LN}$ and subcarinal LN was $>1.0 \mathrm{~cm}$ on contrast-enhanced chest $\mathrm{CT}$, which was also suspicious for metastatic lymphadenopathy. The other patient had a tumor sized $22.9 \mathrm{~mm}$. Right low cervical and subcarinal LNs had FDG uptake (SUVmax, 2.5 and 4.6, respectively), for which metastatic lymphadenopathy could not be excluded. Final histologic subtypes of pure GGNs in these 2 patients were 2 acinar predominant types in the first case and lepidic type in the second case. The pathologic results of LNs were noncaseating granulomas and chronic granulomatous inflammation, respectively. Abnormal FDG uptake of liver segment 6 (SUVmax, 4.0) was detected in 1 patient, and was confirmed to be negative by dedicated abdominal CT. The sensitivity, specificity, positive predictive value, negative predictive value, and accuracy of PET/CT in detecting metastases were not applicable, $98 \%$ (95\% confidence interval [CI], 94\%-100\%), $0 \%$ (95\% CI, 0\%-71\%), 100\% (95\% CI, 97\%-100\%), and 98\% (95\% CI, 94\%-100\%), respectively. No brain metastasis was found in brain MRI of all 109 patients. The sensitivity, specificity, positive predictive value, negative predictive value, and accuracy of brain MRI in detecting metastases were not applicable, $100 \%$ (95\% CI, 97\%-100\%), not applicable, $100 \% \quad(95 \%$ CI, $97 \%-100 \%)$, and $100 \%$ (95\% CI, 97\%-100\%), respectively. The median follow-up time was 47.9 months, and only 1 patient of $109(0.9 \%)$ who did not have brain metastasis in initial brain MRI developed brain metastasis 30 months after surgical resection of lung adenocarcinoma (tumor size, $18.5 \mathrm{~mm}$; histologic subtype, lepidic).

\section{DISCUSSION}

In the pathologic correlative studies, the area of GGO or the disappearing area on mediastinal window images in high-resolution CT of lung adenocarcinomas corresponds to the pathologically noninvasive area (formerly bronchioloalveolar carcinoma or lepidic component). ${ }^{16-18}$ Many studies have demonstrated that the extent of GGO is an important prognostic factor, with a large area of GGO indicating a good prognosis in lung adenocarcinoma. Furthermore, the percentage of GGO area represents the only independent prognostic factor by multivariate analysis. ${ }^{19,20}$ Consequently, the guideline recommending preoperative conventional staging workup with PET/CT for pure GGN without any solid component is controversial. In this study, neither LN metastasis nor distant metastasis, including of the brain, was found in 164 patients with 175 pure GGO nodular adenocarcinomas, even in cases with a lesion larger than $3 \mathrm{~cm}$ viewed on chest CT scan or pathologic stage T3. Our survival rate was $100 \%$. In the study by Uehara and colleagues, ${ }^{21}$ which had a similar population to our study, the 5-year recurrence-free survival ratio was $98 \%$ in lung adenocarcinoma with GGO ratios of $25 \%$ or more. 
The staging guideline for diagnosis and treatment of lung adenocarcinoma is based on the clinical stage. However, there is no definite guideline for pure GGO nodular adenocarcinomas, especially those $>3 \mathrm{~cm}$ in diameter. We performed preoperative FDG PET/CT as part of the routine evaluation of patients with suspected primary lung cancer. In our study, 136 patients $(83 \%)$ were evaluated using $\mathrm{PET} / \mathrm{CT}$; however, there were no cases of LN metastasis in final pathology. The patients who did not undergo $\mathrm{PET} / \mathrm{CT}$ received contrast-enhanced CT evaluation and had no LN metastasis over a mean period of 47.9 months. These results are supported by previous reports that a PET or PET/CT scan is not required in patients with peripheral stage IA tumors, including pure GGO nodular adenocarcinoma or peripheral $\mathrm{T} 1$ adenocarcinoma. ${ }^{6-8,15,22-27}$ In the study by Chang and colleagues, ${ }^{25}$ among 84 patients who had persistent pure GGN, 10 patients who underwent surgical resection after the lesion increased in size showed no LN metastasis. In another study by Ye and colleagues, ${ }^{27}$ none of the 103 patients with pure GGNs had LN metastasis; in a study by Lim and colleagues, ${ }^{28} 46$ persistent pure GGNs showed no LN metastasis; and in a study by Haruhisa and colleagues, ${ }^{23} 15$ GGNs showed no LN metastasis. Our data and those of related previous studies imply that the diagnostic value of a FDG PET/CT scan in cases of pure GGO nodular adenocarcinoma is more dependent on the density of the nodule than its size, and there is little value in routine preoperative PET scans for pure GGO nodular adenocarcinoma regardless of tumor size, because the scans provide no additional information with respect to management of the disease. In our study, sensitivity of FDG PET/CT and brain MRI were both undefined. Specificity was $97.8 \%$ and $100 \%$, respectively, which indicates that FDG PET/CT and brain MRI are not useful in screening. Positive predictive value was 0 in FDG PET/CT, which indicates low prevalence of metastasis. This also can support potential cost savings, as well as reduction in unnecessary radiation exposure to these patients.

When the International Association for the Study of Lung Cancer/American Thoracic Society/European Respiratory Society first reported the new international multidisciplinary classification for lung adenocarcinoma in 2011, pure GGN was noticed in AIS or MIA. ${ }^{15}$ However, several recent studies have demonstrated that invasive adenocarcinoma may also be pure GGN, even for a histologic subtype of nonlepidic pattern such as well-differentiated or well-organized acinar or papillary-predominant adenocarcinomas. ${ }^{8,15,25,28-30}$ Because of the limited resolution of high-resolution CT images (200-300 mm), stromal or myofibroblastic invasion of $\leq 5 \mathrm{~mm}$ in MIA, or even $>5 \mathrm{~mm}$ in invasive adenocarcinoma, may manifest as pure GGN on high-resolution $\mathrm{CT}^{28,29}$ In our study, among 87 invasive adenocarcinomas, acinar $(\mathrm{n}=23 ; 26 \%)$ or papillary predominant adenocarcinomas $(\mathrm{n}=4 ; 5 \%)$ manifested as pure GGN, and there were no LN metastases in patients with these lesions. More importantly, considering that all of the pure GGNs in the study by Son and colleagues ${ }^{30}$ had no recurrence or LN metastasis during long-term follow-up, ${ }^{30}$ invasive adenocarcinoma manifested as pure GGN on CT scan appears to show relatively less aggressive behavior compared with cases of a part-solid or solid feature on CT scan; thus, PET/ CT adds little value for staging.

In patients with advanced-stage NSCLC, routine imaging of the brain with head MRI (or CT if MRI is not available) is recommended, even in the presence of a negative clinical evaluation. ${ }^{5,6,11,31}$ In 1 study of 29 patients with NSCLC and a primary lesion $>3 \mathrm{~cm}$ in size (ie, TNM stage more advanced than T1 N0 M0), MRI with contrast identified asymptomatic, verifiable metastases to the brain in $22 \%$ of cases. ${ }^{32}$ On the other hand, in the study by Park and colleagues, ${ }^{31}$ brain metastasis was diagnosed in patients with stage I lung cancer as frequently as in those with stages II and III cancer without a statistical difference in the development of brain metastasis according to the pathologic stage. These authors suggest that MRI screening of the brain is necessary in patients with lung adenocarcinoma at all stages. However, the use of routine MRI in the staging of patients with NSCLC and negative clinical evaluations has not been studied adequately. In particular, to our knowledge there is no previous study of the use of preoperative routine brain MRI with pure GGO nodular adenocarcinoma. We performed preoperative brain MRI as part of the routine evaluation of suspected primary lung cancer in 109 patients $(66 \%)$ and found no cases of brain metastasis. According to our results, an initial routine brain MRI is unnecessary in patients with pure GGO nodular adenocarcinoma. However, brain metastasis was found in 1 patient without any symptoms 30 months after the operation. The initial tumor size was $18.5 \mathrm{~mm}$ as stage IA and the pathology was invasive adenocarcinoma with the histologic subtype of lepidic pattern. This case suggests that follow-up brain MRI might be helpful for evaluation of brain metastasis but further studies are needed to determine the optimal time interval of brain MRI.

Surgical resection should be strongly considered when radiologically persistent, pure GGNs are detected that are highly suspicious for primary lung cancer, especially when the lesions are growing or are larger than $10 \mathrm{~mm}$, because size is a well-known risk factor for growth. ${ }^{25,33}$ With regard to the extent of surgical resection, limited resection is acceptable when feasible, even though some GGNs were invasive adenocarcinomas. This is because even when limited resection was performed there were no cases of local recurrence or mediastinal LN metastasis, consistent with previous data. Moreover, patients with pure GGNs had a relatively high probability of second primary GGNs in other lobes, and if these patients were to undergo lobectomy the second surgical resection would 
be limited, especially in cases with central locations. ${ }^{34}$ Recently, an increasing number of studies have reported that limited resection rather than lobectomy is sufficient for the treatment of pure GGN-type primary lung cancer, and systematic nodal dissection may not be required. , $^{8,15,33}$

Our study has several limitations. First, not all patients underwent brain MRI or FDG PET/CT. However, we evaluated metastasis by follow-up CT with or without FDG PET/CT for at least 6 months after the operation. Second, we were unable to make a pathologic diagnosis of $\mathrm{LN}$ in some patients. However, most of these patients had peripheral, small pure GGN without enlarged LNs on chest CT. All of these patients also underwent follow-up chest CT or PET/CT and there was no enlargement of LNs during sufficient follow-up periods. Last, our study was inherently limited by its retrospective design, and may have had a selection bias. Therefore, further prospective studies should be performed to evaluate the utility of preoperative FDG $\mathrm{PET} / \mathrm{CT}$ and brain MRI.

\section{CONCLUSIONS}

Neither LN metastasis nor distant metastasis, including brain metastasis, was found in 175 pure GGO nodular adenocarcinomas of 164 patients. We conclude that FDG PET/CT and brain MRI add little to the staging of lung adenocarcinoma manifesting as pure GGO regardless of tumor size, because they provide no additional information with regard to the management of pure GGO nodular adenocarcinoma.

\section{Conflict of Interest Statement}

Authors have nothing to disclose with regard to commercial support.

\section{References}

1. De Leyn P, Dooms C, Kuzdzal J, Lardinois D, Passlick B, Rami-Porta R, et al. Revised ESTS guidelines for preoperative mediastinal lymph node staging for non-small-cell lung cancer. Eur J Cardiothorac Surg. 2014;45:787-98.

2. Rusch VW, Asamura H, Watanabe H, Giroux DJ, Rami-Porta R, Goldstraw P. The IASLC lung cancer staging project: a proposal for a new international lymph node map in the forthcoming seventh edition of the TNM classification for lung cancer. J Thorac Oncol. 2009;4:568-77.

3. Sanchez de Cos J, Sojo Gonzalez MA, Montero MV, Perez Calvo MC, Vicente MJ, Valle MH. Non-small cell lung cancer and silent brain metastasis. Survival and prognostic factors. Lung Cancer. 2009;63:140-5.

4. Koo HK, Jin SM, Lee CH, Lim HJ, Yim JJ, Kim YT, et al. Factors associated with recurrence in patients with curatively resected stage I-II lung cancer. Lung Cancer. 2011;73:222-9.

5. Lee HY, Lee KS, Kim BT, Cho YS, Lee EJ, Yi CA, et al. Diagnostic efficacy of PET/CT plus brain MR imaging for detection of extrathoracic metastases in patients with lung adenocarcinoma. J Korean Med Sci. 2009;24: 1132-8.

6. Silvestri GA, Gonzalez AV, Jantz MA, Margolis ML, Gould MK, Tanoue LT, et al. Methods for staging non-small cell lung cancer: diagnosis and management of lung cancer, 3rd ed: American College of Chest Physicians evidence-based clinical practice guidelines. Chest. 2013;143:211-50.

7. Lee SM, Park CM, Paeng JC, Im HJ, Goo JM, Lee HJ, et al. Accuracy and predictive features of FDG-PET/CT and CT for diagnosis of lymph node metastasis of T1 non-small-cell lung cancer manifesting as a subsolid nodule. Eur Radiol. 2012;22:1556-63.
8. Sim HJ, Choi SH, Chae EJ, Kim HR, Kim YH, Kim DK, et al. Surgical management of pulmonary adenocarcinoma presenting as a pure ground-glass nodule. Eur J Cardiothorac Surg. 2014;46:632-6.

9. Tsutani Y, Miyata Y, Yamanaka T, Nakayama H, Okumura S, Adachi S, et al. Solid tumors versus mixed tumors with a ground-glass opacity component in patients with clinical stage IA lung adenocarcinoma: prognostic comparison using high-resolution computed tomography findings. J Thorac Cardiovasc Surg. 2013; 146:17-23.

10. Howington JA, Blum MG, Chang AC, Balekian AA, Murthy SC. Treatment of stage I and II non-small cell lung cancer: Diagnosis and management of lung cancer, 3rd ed: American College of Chest Physicians evidence-based clinical practice guidelines. Chest. 2013;143:e278S-313S.

11. Hochstenbag MM, Twijnstra A, Hofman P, Wouters EF, ten Velde GP. MR-imag ing of the brain of neurologic asymptomatic patients with large cell or adenocarcinoma of the lung. Does it influence prognosis and treatment? Lung Cancer. 2003;42:189-93.

12. Kim BT, Lee KS, Shim SS, Choi JY, Kwon OJ, Kim H, et al. Stage T1 non-small cell lung cancer: preoperative mediastinal nodal staging with integrated FDG PET/CT-a prospective study. Radiology. 2006;241:501-9.

13. Kim YK, Lee KS, Kim BT, Choi JY, Kim H, Kwon OJ, et al. Mediastinal nodal staging of nonsmall cell lung cancer using integrated 18F-FDG PET/CT in a tuberculosis-endemic country: diagnostic efficacy in 674 patients. Cancer. 2007;109:1068-77.

14. Yi CA, Shin KM, Lee KS, Kim BT, Kim H, Kwon OJ, et al. Non-small cell lung cancer staging: efficacy comparison of integrated PET/CT versus 3.0-T wholebody MR imaging. Radiology. 2008;248:632-42.

15. Travis WD, Brambilla E, Noguchi M, Nicholson AG, Geisinger KR, Yatabe Y, et al. International association for the study of Lung Cancer/American Thoracic Society/European Respiratory Society International Multidisciplinary Classification of Lung Adenocarcinoma. J Thorac Oncol. 2011;6:244-85.

16. Lee HY, Han J, Lee KS, Koo JH, Jeong SY, Kim BT, et al. Lung adenocarcinoma as a solitary pulmonary nodule: prognostic determinants of CT, PET, and histopathologic findings. Lung Cancer. 2009;66:379-85.

17. Okada M, Nakayama H, Okumura S, Daisaki H, Adachi S, Yoshimura M, et al Multicenter analysis of high-resolution computed tomography and positron emission tomography/computed tomography findings to choose therapeutic strategies for clinical stage IA lung adenocarcinoma. J Thorac Cardiovasc Surg. 2011;141:1384-91.

18. Okada M, Tauchi S, Iwanaga K, Mimura T, Kitamura Y, Watanabe H, et al Associations among bronchioloalveolar carcinoma components, positron emission tomographic and computed tomographic findings, and malignant behavior in small lung adenocarcinomas. J Thorac Cardiovasc Surg. 2007;133:1448-54.

19. Ohde Y, Nagai K, Yoshida J, Nishimura M, Takahashi K, Suzuki K, et al. The proportion of consolidation to ground-glass opacity on high resolution CT is a good predictor for distinguishing the population of non-invasive peripheral adenocarcinoma. Lung Cancer. 2003;42:303-10.

20. Takashima S, Maruyama Y, Hasegawa M, Yamanda T, Honda T, Kadoya M, et al Prognostic significance of high-resolution CT findings in small peripheral adenocarcinoma of the lung: a retrospective study on 64 patients. Lung Cancer. 2002; 36:289-95.

21. Uehara H, Tsutani Y, Okumura S, Nakayama H, Adachi S, Yoshimura M, et al Prognostic role of positron emission tomography and high-resolution computed tomography in clinical stage IA lung adenocarcinoma. Ann Thorac Surg. 2013; 96:1958-65.

22. Silva M, Sverzellati N, Manna C, Negrini G, Marchiano A, Zompatori M, et al. Long-term surveillance of ground-glass nodules: evidence from the MILD trial. $J$ Thorac Oncol. 2012;7:1541-6.

23. Matsuguma H, Yokoi K, Anraku M, Kondo T, Kamiyama Y, Mori K, et al. Proportion of ground-glass opacity on high-resolution computed tomography in clinical T1 N0 M0 adenocarcinoma of the lung: A predictor of lymph node metastasis. J Thorac Cardiovasc Surg. 2002;124:278-84.

24. Detterbeck FC, Homer RJ. Approach to the ground-glass nodule. Clin Chest Med. 2011;32:799-810.

25. Chang B, Hwang JH, Choi YH, Chung MP, Kim H, Kwon OJ, et al. Natural history of pure ground-glass opacity lung nodules detected by low-dose CT scan. Chest. 2013;143:172-8.

26. Seki N, Sawada S, Nakata M, Inoue T, Nishimura R, Segawa Y, et al. Lung cance with localized ground-glass attenuation represents early-stage adenocarcinoma in nonsmokers. J Thorac Oncol. 2008;3:483-90.

27. Ye B, Cheng M, Ge XX, Geng JF, Li W, Feng J, et al. Factors that predict lymph node status in clinical stage T1aN0M0 lung adenocarcinomas. World J Surg Oncol. 2014;12:42. 
28. Lim HJ, Ahn S, Lee KS, Han J, Shim YM, Woo S, et al. Persistent pure groundglass opacity lung nodules $\geq 10 \mathrm{~mm}$ in diameter at CT scan: histopathologic comparisons and prognostic implications. Chest. 2013;144:1291-9.

29. Lee HY, Choi YL, Lee KS, Han J, Zo JI, Shim YM, et al. Pure ground-glass opacity neoplastic lung nodules: histopathology, imaging, and management. AJR Am J Roentgenol. 2014;202:W224-33.

30. Son JY, Lee HY, Lee KS, Kim JH, Han J, Jeong JY, et al. Quantitative CT Analysis of Pulmonary Ground-Glass Opacity Nodules for the Distinction of Invasive Adenocarcinoma from Pre-Invasive or Minimally Invasive Adenocarcinoma. PLoS One. 2014;9:e104066.

31. Park HY, Kim YH, Kim H, Koh WJ, Suh GY, Chung MP, et al. Routine screening by brain magnetic resonance imaging decreased the brain metastasis rate following surgery for lung adenocarcinoma. Lung Cancer. 2007;58:68-72.

32. Earnest F, Ryu JH, Miller GM, Luetmer PH, Forstrom LA, Burnett OL, et al Suspected non-small cell lung cancer: incidence of occult brain and skeletal metastases and effectiveness of imaging for detection-pilot study. Radiology. 1999:211:137-45.

33. Naidich DP, Bankier AA, MacMahon H, Schaefer-Prokop CM, Pistolesi M, Goo JM, et al. Recommendations for the management of subsolid pulmonary nodules detected at CT: a statement from the Fleischner Society. Radiology. 2013;266:304-17.

34. Kim HK, Choi YS, Kim J, Shim YM, Lee KS, Kim K. Management of multiple pure ground-glass opacity lesions in patients with bronchioloalveolar carcinoma. J Thorac Oncol. 2010;5:206-10.

Key Words: lung adenocarcinoma, staging, ground-glass opacity, imaging, integrated positron emission tomography/computed tomography, brain MRI

Readers who found these articles interesting may also like to read the following papers found in recent and future issues of our sister publications, Seminars in Thoracic and Cardiovascular Surgery and Operative Techniques in Thoracic and Cardiovascular Surgery!

\section{General Thoracic Surgery: Thoracic Oncology}

News and Views: Novel induction therapies for pleural mesothelioma. Marc de Perrot. Semin Thorac Cardiovasc Surg 2014 Autumn; 26(3):192-200.

State of the Art: Current Innovations in Sentinel Lymph Node Mapping for the Staging and Treatment of Resectable Lung Cancer Yolonda Colson. Semin Thorac Cardiovasc Surg 2014 Autumn; 26(3):201-209

State of the Art: The IASLC/ATS/ERS Lung Adenocarcinoma Classification: What the surgeon should know. Prasad Adusumilli. Semin Thorac Cardiovasc Surg 2014; Autumn; 26(3):210-222.

Current Readings: Percutaneous Ablation for Pulmonary Metastatic Disease. Robert Suh. Semin Thorac Cardiovasc Surg 2014; Autumn; 26(3):239-248.

Current Readings: Window-of-Opportunity Trials for Thoracic Malignancies. Anne Tsao. Semin Thorac Cardiovasc Surg 2014; Winter; 26(4):323-330.

Original Submission: Long-Term Outcomes of Surgical and Nonsurgical Management of Stage IV Thymoma: A Population-Based Analysis of 282 Patients. Masatsugu Hamaji. Semin Thorac Cardiovasc Surg 2015; Spring; 27(1):1-3.

Editorial Commentary: Be Careful about Drawing Big Conclusions from Big Data. Frank C. Detterbeck. Semin Thorac Cardiovasc Surg 2015; Spring; 27(1):4-5.

Original Submission: Surgical Management of Malignant Pleural Mesothelioma: Update of Clinical Evidence. Christopher Cao. Semin Thorac Cardiovasc Surg 2015; Spring; 27(1):6-8.

Original Submission: Retrospective Analysis of Lung Transplant Recipients Found to Have Unexpected Lung Cancer in Explanted Lungs. Takahiro Nakajima. Semin Thorac Cardiovasc Surg 2015; Spring; 27(1):9-14.

Editorial Commentary: A New Set of Lungs or a New Pair of Glasses? Dirk E.M. Van Raemdonck. Semin Thorac Cardiovasc Surg 2015; Spring; 27(1):15-16.

State of the Art: Induction Therapy for Mesothelioma. Isabelle Schmitt-Opitz. Expected publication August 2015.

State of the Art: Precision Therapy for Lung Cancer: Tyrosine Kinase Inhibitors and Beyond. David Schrump. Semin Thorac Cardiovasc Surg 2015; Spring; 27(1):36-48.

Original Submission: Three-hole minimally invasive esophagectomy: a safe procedure following neoadjuvant chemotherapy and radiation. Raphael Bueno. Expected Publication October 2015.

Editorial Commentary: With Minimally Invasive Esophagectomy, Thoracic Surgeons Must Avoid Falling Into the Same Trap Again Thomas Ng. Expected Publication October 2015. 